Vantage: Journal of Thematic Analysis

ISSN: 2582-7391

A Multidisciplinary Publication of Centre for Research, Maitreyi College, University of Delhi

October 2020, Volume 1, Issue 2

Invited Article

\title{
Revisiting the Relationship between Health and Environment during Pandemic Times
}

\author{
Balaganapathi Devarakonda ${ }^{1 *}$ and Saathvik Devarakonda ${ }^{2}$ \\ ${ }^{1}$ Department of Philosophy, University of Delhi, Delhi \\ ${ }^{2}$ Aravind Gupta Anglo Vedic Centenary Public School, Delhi \\ *Correspondence: balaganapathid@gmail.com
}

\begin{abstract}
Health and environment are the basic conditions of human life that are intricately associated with each other. An appropriate exploration into their intricate relationship will reveal that they are not just conditions of human life, rather they fundamentally constitute the life of human beings. This article, explores this relation from varied perspectives including that of the Covid-19 pandemic. The first part of the article argues that the definitions of health have to extend their scope to environment as well, considering the life to be an interrelated and integrated one where healthy environment is a necessary condition for the health of any of its elements or beings. In the second part, the distinction and distinctive relation between two natural calendars that determine the life on earth such as calendar of health and calendar of longevity is examined to show the natural harmony between health and environment. In this regard with the help of the concept of autophagy that was explained in terms of biological cells by Yoshimori Ohsumi, a Japanese Nobel Laureate, it is argued that Covid-19 pandemic has occasioned an accidental solution to the environmental problems in the form of human lockdown that helped the nature to recoup and rejuvenate itself.
\end{abstract}

\section{Exploring the relation}

By referring to health we often limit ourselves to the individual human biological condition. This reductionist approach to health is based on the biomedical approach which underlines a state of normal function of the body that may get affected by disease time to time. This reductionist perspective was corrected by World Health Organisation (WHO) in its definition that, "Health is a state of complete physical, mental and social 
well-being and not merely the absence of disease or infirmity. (1948)" This definition not only corrected the limitations of the reductionist approach to health, rather it widened the scope of health beyond physical to psychological and social spheres as well. However, even the definition of WHO is limited in the sense that it didn't take the environment into consideration. Consequently, it is endowed with at least two limitations: one, the inability to understand the importance of the environment in determining the health of the individual; two, the inability to recognise the importance of the health of the environment which plays a crucial role in the sustenance of the human health for generations. These two are crucial to the healthy life on earth, whether it is of a human being, or a plant or an animal or the elements of nature.

We can infer from the above discussion that a) health is not the sole prerogative of human beings alone, but other beings and elements of life and nature also have health; b) health of the environment is the basic requirement of the health of the human beings. Consequently, it must be understood that plants, animals and even the elements of nature (such as earth, water, fire, air etc.,) which constitute the environment also have health. Unless the health of each one of these is maintained, no one, including human beings, can be guaranteed with a healthy life. It is necessary to understand that health is an interdependent and holistic form of existence. For instance, if your hand is infected you can't claim to be healthy, as it is an integral part of your body, similarly if the health of the one of the elements of the nature is at stake, you can't be said to be maintaining health. Understanding of this integration with the environment and attempting to live in harmony with it is the necessity of the holistic conception of life.

Recognising the importance of environment and understanding its crucial role in determining the health of the humans requires an in depth analysis of the relationship between the two. There are two pointers which are important with respect to the life on earth; first is health and the second is longevity. Let me discuss these two pointers in detail.

\section{Calendars of Health and Longevity}

Human life is often understood in terms of calendars; calendars that are developed to regulate various activities of human life. Though there are many variations in the 
conceptualisation and adaptation of them in various cultures, calendars are primarily invented to regulate human activity.

We come across various calendars such as Gregorian, Julian, Hindu, Islamic, Jewish, Persian, Chinese, Mayan etc., that explicate the attempt of the cultures and civilisations in regulating the human activity. This regulation is often understood to be limited to external activities of the people of that culture or civilisation. These activities include social, political, agrarian, religious etc. that constitute the external life of humans encapsulated in the empirical world. Some of the calendars are based on the movement of Sun and Moon.

It must be noted in this context that most of the classical calendars of various civilisations give significant importance to bring a pause in human activity whether it be eating, or working - in terms of fasting and not speaking for a day or restricting the human activity to home or temples for some time etc.

Along with the artificial ones that humans invented, there are natural calendars as well. It is necessary to note that every element of nature has at least two calendars; the calendar of life and the calendar of health. To explain it scientifically, the former is concerned with longevity whereas the latter is rooted in the capacity to act within which the process of degeneration and recuperation is imbued. It must be noted that the process of degeneration and recuperation largely determines the longevity. Though degeneration is an integral part of natural process, stretching the capacity of activity diminishes the possibility of rejuvenation and effects the health calendar intensely and thus adversely affects the life calendar of any natural element.

Autophagy is precisely the solution for rejuvenation. The process of recuperation by consuming the damaged or degenerated assemblages is understood to be autophagy, a health calendar, with reference to cells. A fixed period of fasting has been prescribed in most of the cultures around the globe according to the geographical and climatic conditions of the place where the culture is rooted. The study of Yoshimori Ohsumi, a Japanese cell biologist who won the Noble prize for Medicine in 2016, argued that a certain duration of time is required for the cells to rejuvenate and heal themselves (1992). This duration must be made possible by pausing intake (consumption of food). 
"During autophagy, it is explained, cells get rid of damaged structures which is crucial for the health, renewal, and survival of cells. Through his research, Ohsumi proved that what activates autophagy in human cells to recycle and renew their content is fasting. How can pausing food intake contribute to the recuperation of the human cell? This is possible because the health calendar of the cell requires that it should not be fed further so that it consumes its damaged structures and thus makes space to rejuvenate. This is the basic principle of recuperation in nature, a calendar of health.” (Devarakonda \& Devarakonda, 2020) This calendar of health largely determines that calendar of longevity of cells as well.

This cell calendar is a scientifically proven one. Every element of nature may have such calendars which have to be discovered. Natural elements may consume their damaged structures, rejuvenate their health and begin to work with their optimum capacity, if they are provided with sufficient space. This space is to be created by pausing or halting the human activity. Through inactivity human beings can trigger the process of autophagy in nature that may affect both the health and longevity calendars of the human beings as well as the environment positively.

The global Covid'19 pandemic had necessitated a complete lockdown in most of the parts of the world that paused all human activity. Due to the pause in human activity all around the globe, there emerged an accidental solution for the so called irreparable problems of the environment that have been troubling the people since past few decades. Ganges, a holy river in India is known to be polluted for a long time. It was in the 1970's that everyone started talking about pollution of the Ganges and thousands of crores of rupees were sanctioned for the sake of cleaning the river since 2016, yet, no improvement was noticed. On 24th of March, 2020, when a nation-wide lockdown was announced by the Government of India, there was a complete pause of human activity. Within a span of less than two months, a river which was known to be polluted for almost half a century revived itself in an astonishing manner. ${ }^{1}$ In the same way, there

1 Kriti Mehta in "Ganga River before and after lockdown: 5 facts you should know" informs us that "Sushant Mishra, chief of Sanga Seva Nidhi in Varanasi told the Quint that the level of water in Ganga has risen, its quality improved, and the dissolved oxygen (DO) in the water has increased too. 
has been similar improvement in problems like depletion of Ozone layer, water and air pollutions caused by human activity. It has been proved through decades of studies on Pollutions and other problems that human activity is the major cause.

One of the indirect and unanticipated consequences of the lockdown is self-healing, recouping, and re-invigoration of the natural environment. A pause in the human activity provided sufficient space for self-curing of nature which has been persistently damaged due to excessive human intrusion.

To sum up, human life is essentially constituted by health and environment both of which are intricately related and interdependent. The definition of health should include environment in its fold and improvement of environment must be understood in terms of the health. Healthy life thus includes the health of each of the elements of environment without the exclusion of human beings. Similarly healthy human life is possible only within the ambit of healthy environment. This interdependency and harmony is very important to have a holistic conception of human life. Health, whether it is of human beings or environment, it is argued, can only be maintained by providing sufficient space for their rejuvenation. This is possible only with a pause in the human activity. That is how it is required to study the health calendars of all the elements of nature in order to correlate them with that of the human activities.

\section{REFERENCES}

Devarakonda, B., \& Devarakonda, S. (2020). Global Lockdown Calender. Philosophical Practice, 15, 2485-88.

Mehta, K. (April 28, 2020). Ganga River before and after lockdown: 5 facts you should know. Retrived from https://www.timesnownews.com/mirror-now/infocus/article/ganga-river-before-and-after-lockdown-5-facts-you-shouldknow/584305

The quint report said that in Varanasi's Nagwa Nala, the measure of DO increased from 3.8 ML/L on March 6 to $6.8 \mathrm{ML} / \mathrm{L}$ on April 4, which enhances the natural cleansing mechanism of the water." 
Takeshige, K., Baba, M., Tsuboi, S., Noda, T. \& Ohsumi, Y. (1992). Autophagy in Yeast Demonstrated with Proteinase-Deficient Mutants and Conditions for its Induction. The Journal of Cell Biology.119(2): 301311.doi:10.1083/jcb.119.2.301.

Tobías, A., Carnerero, C., Reche, C., Massagué, J., Via, M., Minguillón, M. C., Alastuey, A., \& Querol, X. (2020). Changes in air quality during the lockdown in Barcelona (Spain) one month into the SARS-CoV-2 epidemic. The Science of the total environment, $726, \quad 138540$. https://doi.org/10.1016/j.scitotenv.2020.138540.

How to cite this article: Devarakonda, B. \& Devarakonda, S. (2020). Revisiting the relationship between Health and Environment during Pandemic times. Vantage: Journal of Thematic Analysis, 1(2): 6-11.

DOI: https://10.52253/vjta.2020.v01i02.02

(C) The Author(s) 2020.

This work is licensed under a Creative Commons Attribution 4.0 International License which permits its use, distribution and reproduction in any medium, provided the original work is cited. 